\title{
Study of a Gateway Architecture for Wild Animal and Plant Monitoring
}

\author{
Jaehong RYU, Naesoo KIM and Kyesun LEE \\ Electronics and Telecommunications Research Institute, Korea
}

Dongwon KIM

Chungbuk Province University, Korea

\begin{abstract}
Recent changes in the ecosystem and the variations in pollutants will soon be a major threat to the human race. In order to address this issue, predicting changes of the ecosystem based on real-time habitat monitoring is an important and essential technology. Recent advances in wireless sensor network technologies provide us with a chance to be apply ecosystem monitoring to various environments. Nevertheless, there is a need for an application specific gateway architecture, which allows effective inter-working with low-power sensors and an application server on the Internet. In this paper we present details on the application domain of wild animal and plant monitoring along with a gateway architecture for such applications.
\end{abstract}

KEYWORDS: USN; gateway; sensor; low-power

\section{INTRODUCTION}

With the start of the 21 st century, ecosystem changes and environment pollutions arise as one of the greatest threat that we have to overcome. To maintain a sustainable society, we need a long-term and systematic plan in developing systems for ecosystem management and change prediction. In the United States, more than 1.5 million environment monitoring sensors and about 11,700 climatological stations are already in operation. However, in Korea, the Korea Global Atmosphere Watch Center and the only operational center and the domestic production rate of these sensor's are below 13\%. [1]

In order to monitor the environmental ecosystem, communication, surveillance, and control methods should be optimized with respect to the characteristics of target ecosystem. As an example, different systems can be optimal for environments that deal with animals, plants, soil, water, and atmosphere, respectively. Furthermore, previous static monitoring stations can only cover a small geographical area, making it difficult to monitor large regions such as lakes, roads, or forests. Luckily, wireless sensor networks (WSNs) is one of the ideal solutions for wild animal and plant monitoring. Nevertheless, the system designer takes the responsibility to carefully consider the environmental characteristics for not only the communication media, but also to maintain a long lifetime and robust operations under various circumstances.

In this paper, we present a wireless sensor networks structure for wild animal and plant monitoring. Especially, to overcome the aforementioned problems, we suggest a gateway structure which can manage different types of wild animal and plant monitoring systems. Never use letterspacing and never use more than one space after each other.

\section{SENSOR NETWORK STRUCTURES FOR WILD ANIMAL MONITORING}

Figure 1 shows one of our application scenarios for monitoring wild animals' locations based using WSNs. In this scenario, the WSN contains a positioning tag attached on the animal, a low-power sensor node with a fixed position, and a gateway [2],[3].

Recently, the Global Positioning System (GPS) is one of the best positioning technologies for an outdoor-operating system. However, the GPS signal cannot reach some regions in the forest and mountain areas. As a restful a wildlife monitoring system would have to collaborate other positioning technologies with the the GPS' rough estimations. In the case of cellular networks, the cell coverage in the mountain area is over hundreds of meters which makes it un-suitable for wild animal tracking application requirements. To track wild animals, we 
suggest the use of a the hybrid method, which uses not only the GPS and cellular networks, but also low-power sensor nodes which have fixed positions. These nodes help cover the localization functionalities in the shading areas of GPS and cellular network services. Moreover, the static sensor nodes can equip various sensors to collect the animal's habitat data simultaneously. These lowpower nodes connect themselves to a gateway node and the gateway finally forwards wild animals' position data and habitat sensing values to a realtime ecosystem server, for predictions and management, using back-end communication methods.

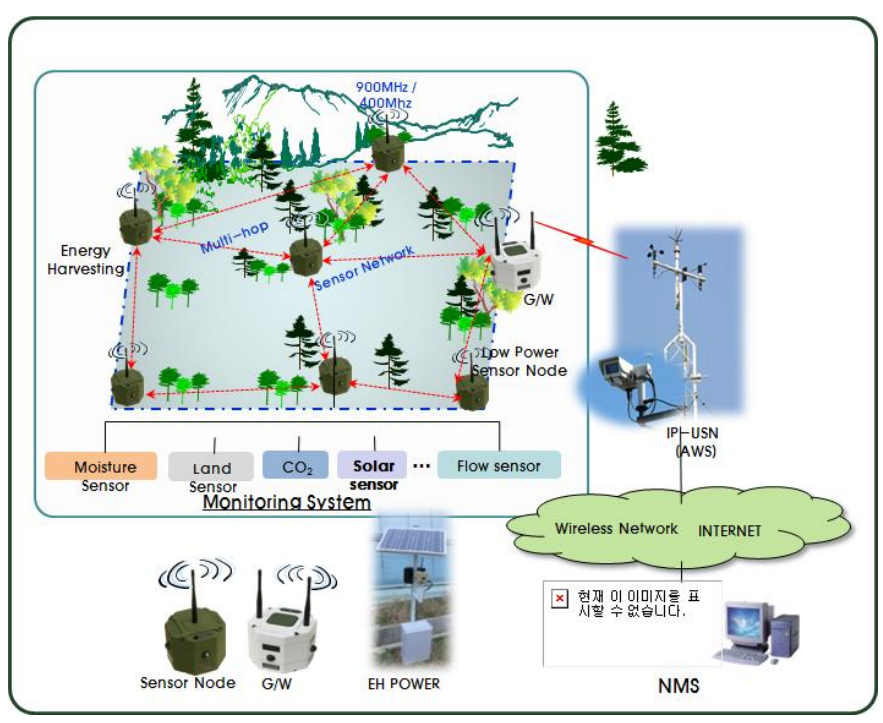

Figure 1. WSN-based Monitoring System

\section{GATEWAY STRUCTURE}

Basically, the gateway functions as a sink node in a typical WSN. In other words, the gateway collects all the sensing values from the sensors and forwards them through an Ethernet or CDMA connection to a back-end server. The hardware architecture of the gateway should be different from sensor nodes to maintain the networks, bridge different communication media, and process sensing values. This implies that the gateway needs a different energy saving method to achieve long lifetimes in the target field. Based on our preliminary investigation, wild animals may not move fast and frequent under the rain. On the contrary, soil, temperature, humidity, or other sensing values, which relates to plants, shows different characteristics when raining. Therefore there is a need to carefully collect data during the raining periods. In order to adaptively control the data reporting duration (also as a way to conserve energy), we attached a rainfall sensor to the gateway. The results from this sensor is used to control the interval of data collection, low rates when there is no rain with increasing rates with increasing rainfall.

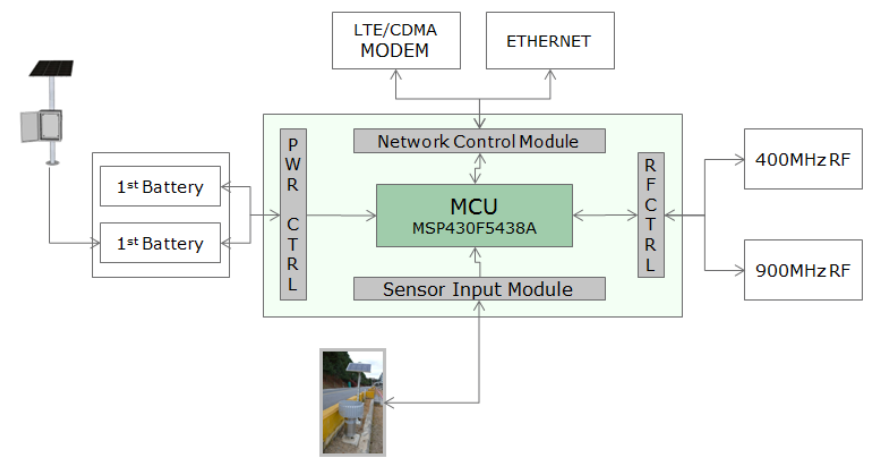

Figure 2. Gateway Systems Architecture

The power control module in the gateway contains both a solar-cell based secondary cell and a battery. When possible, the secondary cell provides a power in most cases, and the battery is only switched as a supplementary power source when the secondary cell is running low on charge.

\section{a) Hardware}

- The gateway includes a 400/900MHz band radio for sensing values collection, and an ethernet or a CDMA module to enable communications with the remote monitoring system.

- Main board: MCU, power control module, 400/900MHz band radio

-Extension board: Ethernet/CDMA, camera module

b) Software

- HAL: Abstract, recognize, and control the hardware

- Core software: MAC and Network layer software for network initialization and routing, CDMA forwarding

- Rainfall sensor-based reporting rate adaptation software

- Power control software for an effective use of the battery and energy harvesting module

\section{CONCLUSION}

In this work, we present a gateway architecture for real-time ecosystem monitoring. The gateway takes the role of forwarding packets from the WSN deployed in the target field to an application's backend server. The proposed architecture takes in consideration the low-power constraints of outdoor WSNs by utilizing environmental factors such as the rainfall rate to determine the sensor reporting intervals. We envision that the same gateway design can be applied to various real-time ecosystem monitoring applications such as wild animal and plant monitoring.

\section{ACKNOWLEDGEMENTS}

This work was supported by Ministry of 

Environment of ROK project number
2013000210002

\section{REFERENCES}

[1] N.Kim, B.Choi, J.Ryu, "A Realtime ecosystem monitoring low power sensor network architecture." Korean Conference on Life Sciences, 2013.
[2] J.Lee, B.Choi, N.Kim, J.Oh, "Wild Animal Monitoring System Architecture for Improving Localization and Longevity WSN Operation's, Korean Conference on Life Sciences, 2013.

[3] N.Kim, C.Pyo, C.Oh, E.Kim, "Technology Trends for IoT Convergence Ecosystem Monitoring", Trends in Information Communications Technology, Aug. 2013. 